\title{
Evaluation of Porphyromonas gingivalis in periodontitis patients of Met.S and periodontitis patients without Met.S
}

Ashwini Nareshchandra Walke ${ }^{1 *}$ and Tushar Vitthalrao Bhagat ${ }^{2}$

${ }^{1}$ MDS, Oral Pathologist, Riyadh, Saudi Arabia

${ }^{2}$ MDS, Faculty \& Course Director for Clinical \& Preclinical Fixed Prosthodontics, Department of Prosthetics Dental sciences, College of Dentistry, Prince Sattam Bin Abdulaziz University AlKarj, Riyadh, Kingdom of Saudi Arabia

\begin{abstract}
Oral cavity is said to be the mirror of systemic health. Many systemic diseases first manifest in the oral cavity. Also, an oral disease has a potential to act as an independent risk factor for causing systemic disease. This bidirectional view is gaining acceptance currently, due to findings that, association between periodontal disease and systemic conditions such as Metabolic Syndrome. Effort has brought advances in revealing the etiological and pathological links between the chronic inflammatory dental disease, periodontitis and systemic condition, Metabolic Syndrome. The role of microorganisms in periodontal disease is well documented. In the last 10 years, studies have been published indicating a positive or negative relationship between periodontitis and various systemic diseases, including Met.S. The most frequently isolated microaerophilic pathogens are (A.actinomyctemcomitans, Campylobacter rectus, and Eikenellacorrodens) and anaerobic pathogens are (P.gingivalis, Bacteroidesforsythus, T.denticola, Prevotella intermedia, Fusobacterium nucleatum, Eubacterium, and spirochetes). In the present study P. gingivalis periodontal pathogen confirmed increased colonization of periodontal pathogens, are cultured in both periodontitis patients without Met.S and Periodontitis patients with Met.S.
\end{abstract}

\section{Introduction}

According to WHO Report 2008, In India, 53\% of the deaths occur because of Non-Communicable Diseases (NCD). These NCDs are the result of lifestyle related behavioral risk factors like use of tobacco, physical inactivity, unhealthy diet and harmful use of alcohol leading to metabolic/physiological changes like increased in blood pressure (BP), overweight/obesity, raised blood glucose and raised cholesterol levels. The clinical cluster of hypertension, cardiovascular disease, hyperlipemia, hyperuricemia and type 2 Diabetes had been recognized as syndrome $\mathrm{X}$ which is finally named as metabolic syndrome (Met.S) which was the subjects of this study.

There is a known association between periodontitis and Met.S. The microorganisms associated with periodontitis are diverse. The association between P. gingivalis and periodontitis is well established but the association between $P$. gingivalis and Met.S is not so evident. Studies have also reported presence of $P$. gingivalis in periodontitis, but microbiological status in Met.S patients is not yet evaluated. Hence, with this aim in mind, we conducted the present study to evaluate the incidence of $P$. gingivalis in periodontitis patients with Met.S \& patients with periodontitis without Met.S.

\section{Materials and Methods}

The present study was carried out in the Department of Oral Pathology and Microbiology of Sharad Pawar Dental College. The patients were selected from the Outpatient Department(OPD) of the department of Periodontics, Sharad Pawar Dental College and department of General Medicine, AchayaVinoba Bhave Rural Hospital Sawangi, Meghe Wardha. Before the start of the study, an informed consent was obtained from all the study subjects. The study was approved by the Institutional Ethics Committee, Datta Meghe Institute of Medical Sciences, Wardha (Deemed University) Ref. No.
DMIMS(DU)/ IEC/ 2013-14/ 127 date-30.09.2013.The study was performed on subjects, which were divided into three groups according to their periodontal status and presence or absence of Met.S.

1. Group I: Periodontally healthy subjects without systemic disorder. $(n=50)$.

2. Group II: Chronic Periodontitis patients without Met.S $(n=50)$.

3. Group III: Chronic Periodontitis patients with Met.S $(n=50)$.

The criteria for periodontal disease sites were as follows

1. Probing pocket depth $(\mathrm{PPD})>3 \mathrm{~mm}$, with the help of Williams graduated periodontal probe.

2. Presence of bleeding on probing(BOP).

3. Presence of clinical attachment loss(CAL).

4. Gingival Index score (GI $\geq 2)$.

The criteria for diagnosis of Met.S were as follows

1. Body Mass Index (BMI), Obesity $>30\left(\right.$ mass $^{\mathrm{kg}} /$ height $\left.\mathrm{m}^{2}\right)$.

2. Pressure $(\mathrm{BP})>140 / 90 \mathrm{mmHg}$.

3. Triglycerides (TG) $>160 \mathrm{mg} \%$.

4. Fasting Blood Sugar(FBS) $>125 \mathrm{mg} / \mathrm{dl}$.

5. High -Density Lipoprotein (HDL) $>75 \mathrm{mg} \%$.

${ }^{\star}$ Correspondence to: Ashwini Nareshchandra Walke, MDS, Oral Pathologist, Riyadh, Saudi Arabia, Tel: 091-9975771205; E-mail: ashwiniwalke2210@gmail.com

Key words: Metabolic Syndrome, Periodontitis, Porphyromonas gingivalis

Received: March 24, 2018; Accepted: April 02, 2018; Published: April 05, 2018 
Detailed clinical history was recorded, and clinical assessment was carried out in all the three groups. Subgingival plaque samples were collected and subjected to conventional microbial culture method.

\section{Group I}

Subgingival plaque sample was collected from molar area of periodontally healthy individuals. The sampling site was isolated using cotton rolls and supragingival plaque was removed with the help of sterile cotton. The subgingival plaque sample was then collected using sterile Gracey curettes.

\section{Group II and III}

The sampling sites were isolated with cotton roles and subgingival plaque was removed using sterile cotton. Subgingival plaque samples were obtained using sterile gracy curettes from deepest periodontal pocket. Plaque was subsequently transferred into a sterile container (Himedia) with $0.85 \%$ of sterile saline that was immediately processed. Serial Dilution was done. A serial dilution is the stepwise dilution of a substance in solution. Usually the dilution factor at each step is constant, resulting in a geometric progression of the concentration in a logarithmic fashion. In $100 \mathrm{ml}$ distilled water, $0.85 \mathrm{gms}$ Sodium chloride ( $\mathrm{NaCl}$ - SD fine company) was added. The prepared $9 \mathrm{ml}$ of $0.85 \% \mathrm{NaCl}$ was dispensed into 3 test-tubes (Borosil) each and autoclaved. After autoclaving 5 -fold serial dilutions of the sample was made in the testtube containing $0.85 \% \mathrm{NaCl}$. The serial dilution of samples was done immediately and cultured on agar plates. Brucella Agar Plate with Hemin and Vitamin K1. Hi MP1039 is recommended for the isolation of for $P$. gingivalis. The prepared culture plate was used for inoculation. The plaque sample which was already serially diluted was added to each medium using streak culture method. Streak culture method is a routinely employed method for bacterial isolation in pure culture. A platinum or nichrome wire loop of 2-4 $\mathrm{nm}$ in internal diameter is used. A loopful of specimen is smeared onto the surface of dried plate near the peripheral area. This is known as primary inoculum. From the primary inoculum, it is spread thinly over the plate by streaking with loop in parallel lines. The streak plate technique is essentially a method to dilute the number of organisms, decreasing the density. This allows for individual colonies to be isolated from other colonies. Each colony is considered "pure," since theoretically, the colony began with an individual cell. Each inoculated petri-plate was sealed with Parafilm (Himedia) to prevent any contamination while incubation, and then placed in an anaerobic jar (Himedia) with AnaeroGas Pack system (Himedia) and incubated at $37^{\circ} \mathrm{C}$ for $48-72$ hours. All the plates are then placed in the anaerogas jar and anaerogas pack is placed inside the jar to maintain the anaerobic condition. The anaerobic gas jar is then placed inside the incubator at $37^{\circ} \mathrm{C}$ for 72 hours. After 72 hours the colonies cultured on Petri plates are subjected to biochemical identification test for confirmation of bacteria intended to be cultured. The identification of a bacterial species is based on factors, including colony morphology, chemical composition of cell walls, biochemical activities, and nutritional requirements. 3 points should be considering during microorganism identification. P.gingivalis is gram negative and Indole Test was positive (Table 1).

\section{Observations and results}

In this study, a quantitative analysis of $P$. gingivalis in periodontitis patients with Met.S and periodontitis patients without Met.S done. The results of the present study were subjected to statistical analysis. The comparison of the $P$. gingivalis counts in periodontitis patients with Met.S (Group III), periodontitis patients without Met.S (Group II)
Table 1. Colony Morphology (from agar plates) $P$. gingivalis

\begin{tabular}{|c|c|c|c|c|}
\hline Shape & Elevation & Edge & Color & Surface \\
\hline $\begin{array}{c}\text { Small Rod } \\
\text { shaped }\end{array}$ & Not raised & Irregular & Colourless & Convex \\
\hline
\end{tabular}

and normal control (Group I) was carried out to find the significant difference between those values. The statistical tests used for the analysis of the results were: Chi-square Test, one-way ANOVA (F-Test), Tukey HSD and 'Descriptive statistical analysis' (i.e. mean, standard deviation and standard error) was carried out for all the groups in this study.

Among 150 selected patients, $P$. gingivalis were present in 2(4\%) patients of normal group, 31(62\%) patients of periodontitis without Met.S, and $40(80 \%)$ patients of periodontitis with Met.S. Therefore, the results of the study revealed that, $P$. gingivalis is present in descending order from Group III $>$ Group II $>$ Group I. (Table 2 Graph 1).

Among all groups, P. gingivalis were present in 1(50\%) male \& 1(50\%) female patient of normal group, 18(58.06\%) male patients \& $13(41.93 \%)$ female patients of periodontitis without Met.S (Group II), 29(72.5\%) male \& 11(72.5\%) female patients of periodontitis with Met.S (Group III). Presence of $P$. gingivalis in males was more as compared to females in group II \& group III. And there was 2:1 distribution of male:female in Group III. (Table 3).

In Normal controls (Group I), mean total count for P.gingivalis was $0.04 \pm 0.19 \times 10^{5}$ with range of $0.00-1.00$. In Periodontitis patients without Met.S (Group II), mean total count for P. gingivalis was 4.68 $\pm 3.99 \times 10^{5}$ with range of 0.00 - 11.00 . In Periodontitis patients with Met.S (Group III), mean total count for P. gingivalis was $10.94 \pm 0.19 \times 10^{5}$ with range $0.00-20.00$. Thus, statistical analysis revealed that $P$. gingivalis count in periodontitis patients with Met.S was six times more than periodontitis patients without Met.S. In periodontitis patients without Met.S the count was about four times more than normal control. In periodontitis patients with Met.S the count was about eight times more than normal control (Table 4 Graph 2).

The statistically significant variations of mean of total count of P.gingivalis count were found among all groups $(\mathrm{p}<0.05)$. The total counts of $P$.gingivalis were observed in decreasing order in periodontitis with Met.S (Group III) $10.94 \times 10^{5} \pm 5.95 \times 10^{5}$, periodontitis without Met.S (Group II) $4.68 \times 10^{5} \pm 3.93 \times 10^{5}$, normal control(Group I) $0.04 \mathrm{x}$ $10^{5} \pm 0.19 \times 10^{5}$ (Table 5).

The statistical significant difference in total count of $P$.gingivalis was noted between all groups $(\mathrm{p}=0.000)$. Thus, statistical analysis revealed that P.gingivalis count was highest in periodontitis patients with Met.S (Group III) than periodontitis patients without Met.S (Group II) and Normal control (Group I) (Tables 5 and 6).

\section{Discussion}

Oral cavity is said to be an open system which is exposed to the environment. So, the possibilities of foreign material entering the system are heightened because of constant intake of food and liquids through the mouth. Large numbers of bacteria in oral cavity can start tissue destruction by activating on host defense cells indirectly, which in turn, produce as well as release mediators which will stimulate the effectors of connective tissue breakdown.

Met.S refers to a constellation of disturbances including glucose intolerance, central obesity, dyslipidemia (hypertriglyceridemia, elevated nonesterifiedfatty acids (NEFAs), and decreased high-density lipoprotein (HDL) cholesterol, and hypertension. 
Table 2. Presence of P.gingivalis colonies group wise

\begin{tabular}{|c|c|c|c|c|}
\hline Groups & Present & Absent & Total & 2-value \pm SD \\
\hline Group I & $02(4 \%)$ & $48(96 \%)$ & $50(100 \%)$ & \\
\hline Group II & $31(62 \%)$ & $19(38 \%)$ & $50(100 \%)$ & $7.55 \pm 1.69$ \\
\hline Group III & $40(80 \%)$ & $10(20 \%)$ & $50(100 \%)$ & $13.68 \pm 2.48$ \\
\hline Total & 73 & 77 & 150 & \\
\hline
\end{tabular}

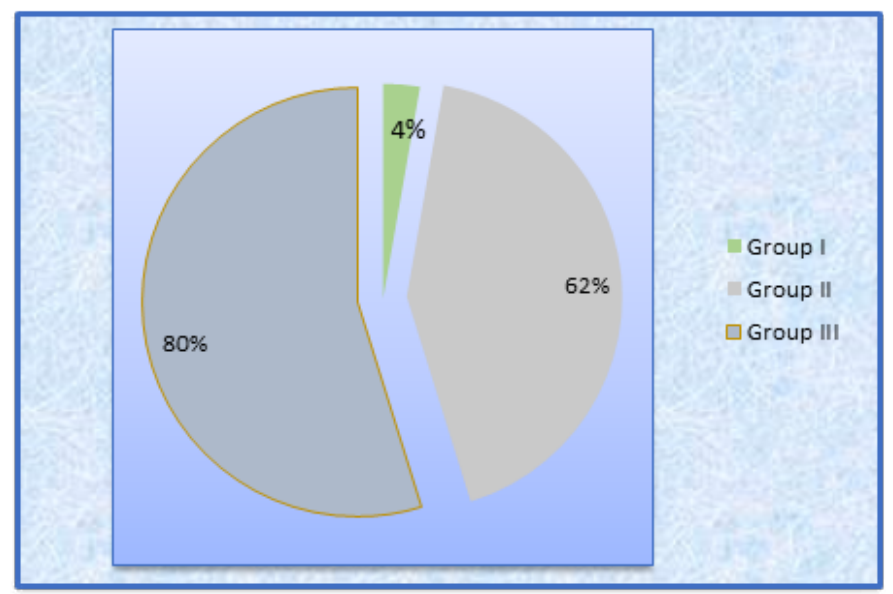

Graph 1. Presence of P.gingivalis colonies group wise

Table 3. Distribution of P.gingivalis according to gender in different groups

\begin{tabular}{|c|c|c|c|}
\hline Gender & Group I & Group II & Group III \\
\hline Male & $01(50 \%)$ & $18(58.06 \%)$ & $29(72.5 \%)$ \\
\hline Female & $01(50 \%)$ & $13(41.93 \%)$ & $11(27.5 \%)$ \\
\hline Total & $02(100 \%)$ & $31(100 \%)$ \\
\hline
\end{tabular}

Table 4. Descriptive Statistics of P.gingivalis count in all three groups. (All the values in $10^{5}$ )

\begin{tabular}{|c|c|c|c|c|c|c|c|}
\hline \multirow{2}{*}{ Groups } & N & Mean & \multirow{2}{*}{ Std. Deviation } & \multirow{2}{*}{ Std. Error } & \multicolumn{2}{|c|}{ 95\% Confidence Interval for Mean } \\
\cline { 3 - 7 } & & & \multirow{2}{*}{ Lower Bound } & Upper Bound & Minimum & \multirow{2}{*}{ Maximum } \\
\hline Normal & 50 & 0.40 & 0.19 & 0.027 & -0.01 & 0.09 \\
\hline Periodontitis without Met.S & 50 & 4.68 & 3.93 & 0.55 & 3.56 & 1.00 \\
\hline Periodontitis with Met.S & 50 & 10.94 & 5.95 & 0.84 & 9.24 & 11.00 \\
\hline
\end{tabular}

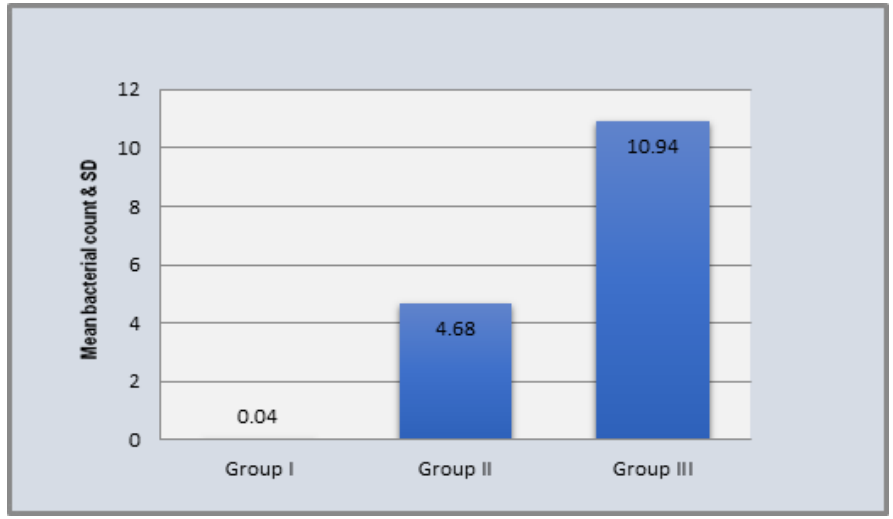

Graph 2. Comparison of total count for P.gingivalis in three groups. (All values in $10^{5}$ )

Table 5. One-way ANOVA for P.gingivalis count in all three groups. (All the values in $10^{5}$ )

\begin{tabular}{|c|c|c|c|c|}
\hline Source ofvariation & Sum of Squares & Df & Mean Square & p-value \\
\hline Between Groups & 2992.12 & 2 & 1496.06 & 16.97 \\
\hline Within Groups & 2495.620 & 147 & 0.000 \\
\hline Total & 5487.74 & 149 & 88.123 \\
\hline
\end{tabular}


Table 6. Multiple Comparisons: Tukey Test for P.gingivalis count in all three groups (All the values in $10^{5}$ )

\begin{tabular}{|c|c|c|c|c|c|c|}
\hline \multirow{2}{*}{\multicolumn{2}{|c|}{ Groups }} & \multirow{3}{*}{$\begin{array}{c}\text { Mean Difference (I-J) } \\
-4.64\end{array}$} & \multirow{3}{*}{$\begin{array}{c}\text { Std. Error } \\
0.82\end{array}$} & \multirow{3}{*}{$\begin{array}{c}\text { p-value } \\
0.000 \mathrm{~S}, \mathrm{p}<0.05\end{array}$} & \multicolumn{2}{|c|}{ 95\% Confidence Interval } \\
\hline & & & & & \multirow{2}{*}{$\begin{array}{c}\text { Lower Bound } \\
-6.59\end{array}$} & \multirow{2}{*}{$\begin{array}{c}\text { Upper Bound } \\
-2.68\end{array}$} \\
\hline \multirow{2}{*}{ Normal } & Periodontitis without Met.S & & & & & \\
\hline & Periodontitis with Met.S & -10.90 & 0.82 & $0.000 \mathrm{~S}, \mathrm{p}<0.05$ & -12.85 & -8.94 \\
\hline Periodontiti patients with Met.S & Periodontitis without Met.S & 6.26 & 0.82 & $0.000 \mathrm{~S}, \mathrm{p}<0.05$ & 4.30 & 8.2 \\
\hline
\end{tabular}

In our study, periodontitis patients with Met.S has higher predilection for male patients, the reasons for this findings could be, habits like smoking, pan chewing with/without tobacco which contribute to poor oral hygiene leading to periodontal diseases more commonly, Along with the above mentioned reasons, the male patients of Met.S had higher BP, increased serum levels of triglycerides, higher insulin sensitivity, and more abdominal fat accumulation than females therefore increasing the chances of development of periodontal diseases in male patients. The results of our study are in accordance with Han DH etal 2010. However, higher inflammation in females with Met.S than in males, could be a-related factor associated with periodontal disease in females than in males.

Another reason for the gender difference in the association between periodontal disease and Met.S may be the role played by sex hormones estrogen in the inflammatory process. Estrogen is the primary hormone responsible for development and regulation of female reproductive system. Insulin has a pivotal role in facilitation of anabolic processes and in activation of transport system being associated with metabolism of carbohydrate and lipids. It is also acts as a potent regulator of human sexual hormone synthesis and interferes with their signal transduction at cellular level, which was observed in Met.S. Insulin and estrogen have protective mechanisms in younger females, whereas in males there is a deficiency of this combination. There has been a documented association of decreased IR with increased risk of CVS disease, along with severity of periodontal infection in cases of metabolic syndrome. Therefore, considering all these factors, it could be hypothesized that males showed high predominance of periodontitis in Met.S patients.

The microbiological analysis revealed that the mean count for P.gingivalis in periodontitis patients was six times more than control. The results of our study were in an agreement with the study of Ann L. Riefenstahl, which showed that P.gingivalis were present in low levels in healthy subjects and increased 11.2-fold in individuals with periodontitis.

The reasons for increased colonies of P.gingivalis in periodontitis patients could be that, $P$. gingivalis is best characterized as an opportunistic oral pathogen that inhibits the oral biofilm. Many virulence mechanisms have been identified for P.gingivalis. P.gingivalis has a carbohydrate capsule on its outer surface which prevents opsonization by complement and inhibits phagocytosis and killing by neutrophils. The lipopolysaccharide which is produced by it is not very strong, but it could inhibit chemotaxis and killing by leucocytes. This organism possesses several putative virulence factors (including proteases which degrade immunoglobulin, complement, collagen fibres, hyaluronic acid; adhesins, endotoxins, and cytotoxins) that can directly affect the periodontium or elicit host functions that result in the periodontal disease.

The results of our study revealed that mean count for P.gingivalis in periodontitis patients with Met.S was four times more than periodontitis patients without Met.S and 8 times more than controls. Our results are in accordance with the study results by Takahashi etal, 2001 who states that levels of P.gingivalis was increased in periodontitis patients with Met.S as compared to normal patients.
The reasons for increased in P.gingivalis levels in periodontitis patients with Met.S could be explained as, P.gingivalis, have the known ability to invade deep vascular endothelium of the periodontium of Met.S patients comprising of T2DM. Once periodontal pathogens were entered and established in the diabetic host, periodontal infection may aggravate microvascular complications. In periodontitis patients, chronic low-level systemic exposure to periodontal microorganisms exists, that will lead to significant changes in plasma levels of cytokines and hormones. Due to the dynamic nature of the inflamed periodontium, the tissue may act as an endocrine-like source of inflammatory mediators. Among the inflammatory biomarkers, TNFalpha, CRP, IL-1 $\beta$, IL- 6 and IL- 8 and IL- 6 are liberated. Excessive concentrations of TNF-alpha negatively regulate insulin signaling and glucose uptake. Additionally, the elevated levels of soluble TNF receptor 1 and 2 (sTNFalphaRI and sTNF-alphaRII) shown in obese patients may lead to a hyperinflammatory state, thereby increasing the risk for periodontal disease. The hyperinflammatory state may be caused by adipocytes, which secrete proinflammatory cytokines, and providing the link between the pathogenesis of type 2 diabetes, obesity, and periodontal disease. The highest levels of TNF-alpha and sTNFalpha receptors were found in those individuals in the highest quartile for BMI. These observations may suggest, an increased acute phase response is associated with insulin resistance contributing to periodontitis.

One of the complications of diabetes is elevation of blood glucose concentrations (hyperglycemia). Hyperglycemia leads to the formation of AGEs. These AGEs make endothelial cells and monocytes more susceptible to stimuli that will then induce the cells to produce inflammatory mediators. Some author suggested that AGE accumulations in the gingival tissue will leads to increased vascular permeability, and increased breakdown of collagen fibers, and more destruction of both nonmineralized connective tissue and mineralized bone. As diabetes is a metabolic disorder and periodontal disease is an infectious disease, the pathophysiological relationship between these two entities is, through the ability of both conditions to induce an inflammatory response, because of AGE or bacterial accumulation, respectively, which further leads to the production of inflammatory mediators.

\section{The following conditions in Met.S favor the growth of P.gingivalis}

P.gingivalis attached to the components of extracellular matrix and to the Type I collagen. This helps in colonization of P.gingivalis to gingival margin. The arg-gingipain protease of P.gingivalis helps either directly or indirectly in this attachment. Directly by acting as an adhesion and indirectly by affecting the expression of an adhesion. A strong association between gingival protease activity and periodontal infection were found in studies. In Met.S serum levels of Cystatin C (cysteine protease) are synthesized at constant rate. Therefore, it could be hypothesized that constant increased in protease in Met.S patients favours the colonization of P.gingivalis. Other reasons for increase colonization of P.gingivalis could be that P.gingivalis can identify receptors on gingival epithelial cell receptors even in low chronic inflammatory condition, P.gingivalis also has the property to respond and adopt to oxidative stress as they are oxidative stress resistance. P.gigivalis growth requirements include iron, which is provided by elevated iron stores in GCF of Met.S patients. 
Thus, Patients diagnosed with Met.S are thought to be at higher risk due to a compromised immune system. Infectious and opportunistic microbes responsible for periodontal infection may thus bring a burden onto the rest of the body and release products that elicit an inflammatory response.

\section{Conclusion}

The fact that increases in colonization of periodontal pathogens may be attributed to the assumption that, Periodontitis releases proinflammatory cytokines and ROS at the site of inflammation, leading to oxidative stress situation. This will contribute to aggravate existing Met.S.

\section{Acknowledgment}

The first author would like to acknowledge Dept. of Oral Pathology and Microbiology, Dept. of Periodontics, Sharad Pawar Dental College and department of General Medicine, Achaya Vinoba Bhave Rural Hospital Sawangi, Meghe Wardha.

Copyright: $\odot 2018$ Walke AN. This is an open-access article distributed under the terms of the Creative Commons Attribution License, which permits unrestricted use, distribution, and reproduction in any medium, provided the original author and source are credited. 